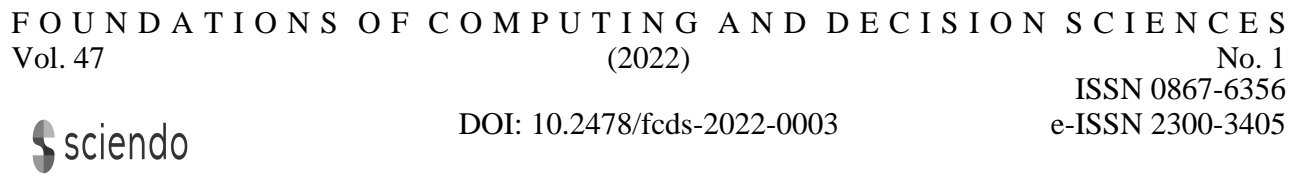

\title{
ReqTagger: A Rule-Based Tagger for Automatic Glossary of Terms Extraction from Ontology Requirements
}

\author{
Dawid Wiśniewski, Jędrzej Potoniec, Agnieszka Ławrynowicz *
}

\begin{abstract}
Glossary of Terms extraction from textual requirements is an important step in ontology engineering methodologies. Although initially it was intended to be performed manually, last years have shown that some degree of automatization is possible. Based on these promising approaches, we introduce a novel, human interpretable, rule-based method named REQTAGGER, which can extract candidates for ontology entities (classes or instances) and relations (data or object properties) from textual requirements automatically. We compare REQTAGGER to existing automatic methods on an evaluation benchmark consisting of over 550 requirements and tagged with over 1700 entities and relations expected to be extracted. We discuss the quality of REQTAGGER and provide details showing why it outperforms other methods. We also publish both the evaluation dataset and the implementation of REQTAGGER.
\end{abstract}

Keywords:Competency Questions, Ontology Requirements, Ontology, Information Extraction, Part-of-Speech tagging

\section{Introduction}

Ontologies are formal representations of knowledge, describing a given domain of interest in terms of entities and relations between them. They are widely used to model various domains like: Internet of Things, biology, medicine or music. Ontologies are usually expressed using logic-based languages such as OWL [1], enabling them to express non-trivial relations between entities and allowing to use reasoning to infer new facts from existing knowledge. The possibilities given by logic-based languages, however, make the ontology construction process (ontology authoring) a challenge. Ontology engineers have to ensure that all required entities and relations are modelled in an ontology and make sure that the logical conclusions of modelling

\footnotetext{
*Faculty of Computing and Telecommunication, Poznan University of Technology, Poland, \{dawid.wisniewski@cs.put.poznan.pl
} 
decisions are correct. For this reason, many ontology engineering methodologies were proposed, sharing the common goal of trying to structure the process of ontology creation and maintenance. Ontologies are defined for various purposes and used in diverse scenarios. Some of them model common knowledge while other model specific domains. Some are expected to be complete in a fixed period of time, while others are intended to be continuously updated. Also, some ontologies are prepared by only one engineer, while others are constructed collaboratively. This variety of needs and scenarios resulted in the emergence of multiple methodologies tailored to address challenges faced by projects they were used in. Some of the well known examples are: Methontology [6], NeON [3], OnToKnowledge [23] or TDDOnto [12]. Many of the methodologies require an upfront formulation of so-called requirements, stating what the ontology should model in a natural language, either as questions, called competency questions (CQs) or as statements. An example CQ What software works on Linux? indicates that software and Linux entities as well as works on relation must be modelled in a given ontology to make the CQ answerable.

Requirements are used for multiple reasons, two of which we find of high importance:

1. To check if the ontology being modelled can be considered complete by verifying if the ontology can answer all competency questions correctly.

2. To provide an overview of which entities and relations should be modelled in the ontology.

In the NeON methodology, CQs are used in the process of extraction of so-called Glossary of Terms, providing all entities (classes and instances) and relations (object and data properties) stated in a CQ set. The Glossary of Terms is a part of Ontology Requirements Specification Document. Although the process of Glossary of Terms extraction was designed to be manual, in the last years some ideas to automate the process have emerged, giving us hope that the process of ontology engineering will be quicker and less complicated for engineers. In this paper, we automate the Glossary of Terms extraction process using a novel rule-based system called REQTAGGER.

The contributions of this work are as follows:

- REQTAGGER - a rule based system for extracting candidates for ontology entities from ontology requirements automatically.

- An evaluation dataset of requirements tagged with entities and relations to be extracted.

- An evaluation comparing REQTAGGER to other automatic methods.

In this work we address the following research questions:

- RQ1: Does a rule-based method implemented in REQTAGGER provide better quality of automatic Glossary of Terms extraction than the existing alternatives?

- RQ2: Does the method generalize from requirements expressed as questions to requirements expressed as statements? 
The rest of the paper is structured as follows: we cover related work in Section 2, describe materials used to construct and evaluate the method in Section 3, provide a REQTAGger description in Section 4. We evaluate the method in Section 5, discuss the errors in Section 6, present the evaluation scores and answer research questions in Section 7, discuss the limitations of REQTAGGER in Section 8 and conclude in Section 9 .

\section{Related works}

Ontology engineering methodologies The first attempts to formalize ontology engineering methodologies can be traced back to the CYC project by Lenat et al. [13]. CYC authors introduce general recommendations (e.g., one should model abstract knowledge first, before modelling domain knowledge) to improve the quality of new ontologies. Uschold and King [24] presented how the ontology development process can be split into separate phases: purpose identification, ontology building, evaluation and documentation. Gruninger and Fox [8] noticed the need for specification of the motivating scenarios of the ontology usage. These can be treated as sources of competency questions that define a set of requirements a completed ontology should meet. They are further utilized to assess the completeness of a constructed ontology. Lopez et al. proposed METHONTOLOGY [6], which defines a set of steps used in an evolving prototype scenario, allowing to move back to previous steps in the ontology construction workflow. In METHONTOLOGY, for specification purpose, CQs can be used as an option and Glossary of Terms is required as one of the steps in the conceptualization activity. Suarez-Figueroa et al. introduced NeON [22] methodology, which provides 9 scenarios that can be utilized depending on the engineer's needs, a glossary of processes and activities useful for development purposes, life-cycle models and other processes related methodological guidelines. The glossary of processes defines and classifies required steps, which are named: conceptualization, ontology requirements specification, ontology reuse, ontology evaluation. NeON methodology also provides guidelines on how to create ontologies in a collaborative manner. Sure et al. proposed OnToKnowledge [23], another methodology which puts emphasis on knowledge processes and knowledge meta processes. Similarly to METHONTOLOGY and $\mathrm{NeON}$, OnToKnowledge also suggest creation of a specification of requirements as one of the steps to be performed. During the last years, TDDOnto was introduced by Lawrynowicz et al. [12]. It focuses on assessing quality of knowledge added to the ontology. The main idea behind TDDOnto is to prepare tests first, similarly to common practices from software engineering field, which are then used to check the quality of knowledge added to the ontology.

Requirements collections Requirements being used to construct ontologies are often published along with the ontology itself in the web. In the last years attempts to collect and analyze requirements stated against different ontologies have been made. Wisniewski et al. [26] and Potoniec et al. [20] gathered a dataset called 
CQ2SPARQLOWL ${ }^{1}$ consisting of 234 schema-level competency questions with their formalizations in SPARQL-OWL ontology query language. CQ2SPARQLOWL contains CQs stated against 5 ontologies describing various domains. The main goal of CQ2SPARQLOWL is to analyze how CQs are constructed, what are the similarities between CQs stated against different ontologies and how they can be translated into SPARQL-OWL query language. Fernández-Izquierdo et al. [5] collected even bigger dataset named CORAL ${ }^{2}$ consisting of 834 ontological requirements annotated with lexico-syntactic patterns. In CORAL, 834 requirements stated at the assertion-level and the schema-level are expressed as either CQs or statements. All the requirements come from 14 different ontologies covering domains like: video games, software, health or animals. The aim of this work is to provide a dataset that will ease the automation of requirements into tests. Wisniewski et al. [28] created a synthetic dataset named BigCQ ${ }^{3}$ that consists of 77575 CQ templates mapped to 549 SPARQL-OWL templates. The templates may be filled with labels and IRIs from a given ontology to produce a huge number of CQs and SPARQL-OWL queries. Such a dataset may be used as a silver-standard in the problem of translating CQs into SPARQL-OWL queries automatically.

Patterns in requirements Requirements are often analysed to find recurring patterns which can lead to construction of tools supporting the process of ontology authoring. Keet et al. [10] used CQ2SPARQLOWL dataset to construct a controlled natural language named CLaRO helping engineers to create new CQs. Ren et al. [21] performed an analysis of CQ archetypes (e.g., "Which [CE1] [OPE] [CE2]") containing placeholders for ontology elements like class expressions or object property expressions. They focused on checking how CQs are formulated and how to test if a CQ can be meaningfully answered. Bezerra et al. [2] proposed patterns with placeholders for CQs (e.g. "Is $+<$ class $>+$ a $+<$ class $>$ ?") and used them in a tool named CQChecker to check if existing ontologies meet given requirements. Wisniewski et al. [26] parsed CQs into templates like "What EC1 PC1 EC2?", where ECs represent entity chunks intended to be classes, while PCs represent predicate chunks intended to be relations. ECs and PCs replace ontology vocabulary from CQs, leaving domain agnostic patterns comparable among CQs stated against different ontologies. They were used to check how CQ patterns map to SPARQL-OWL query constructs. Wisniewski et al. [25] proposed an approach to use such patterns in the process of automatic translation of CQs into ontology query language, in order to automatically assess the completeness of a given ontology.

Natural language processing methods for information extraction The task of information extraction from texts is a long existing topic in the field of natural language processing [7]. Most of modern solutions use some form of machine learning, often neural networks [9, 19] or Conditional Random Fields (CRF) [11]. In cases where only a limited size of training data is available, it is important to fuel the model with

\footnotetext{
${ }^{1}$ https://github.com/CQ2SPARQLOWL

${ }^{2}$ https://coralcorpus.linkeddata.es/

${ }^{3}$ https://github.com/dwisniewski/BigCQ
} 
meaningful features. For the task of tagging sequences of words in text (called Named Entity Recognition (NER)) features like part-of-speech tags (POS-tags) assigned to each token, information from dependency parse tree defining relations between tokens, tokens itself or word shapes are frequently used [14].

Automatic Glossary of Terms extraction The automatization of Glossary of Terms extraction was described by Wisniewski et al. in [27], where they presented their tool (further referenced as CRF-BASED TAGGER). They proposed a machine learning-based method, utilizing a CRF model to tag sequences of tokens among CQs which are considered to be good candidates for classes, individuals or properties. They utilized CQ2SPARQLOWL dataset to prepare a tagger that is trained over a subset of CQs stated against the Software Ontology [15] (further abbreviated SWO) and evaluated on the remaining CQs from CQ2SPARQLOWL. Although only 88 CQs are stated against SWO, the dataset size was increased in the process of materialization, as described by Wisniewski et al. [26]. Since many CQs from CQ2SPARQLOWL contain placeholders (e.g. the CQ: What software can perform [task x]? contains a placeholder [task $\mathrm{x}$ ]), the materialization of such CQs can be performed by replacing all placeholders with class labels obtained from the ontology, which meet placeholder restrictions (e.g. replacements of [task x] should be varioius tasks only). As a result 46,465 training CQs were constructed and used for the model training.

Wisniewski et al. [26] used rules and noun chunking to create CQ patterns. They introduced a set of rules based on POS-tag sequences (e.g. the rule $([0-9]+::($ PART |VERB $), ?)+([0-9]+:: \text { VERB })^{4}$ matches at least one verb or particle followed by a verb) to detect so-called predicate chunks. To detect entities (called entity chunks), they used the noun chunker built in $\mathrm{SpaCy}_{\text {tool }}{ }^{5}$. The chunks were later used in order to create domain agnostic CQ patterns. Although they were used in a different context, they can be perceived as rules extracting Glossary of Terms, since they identify domain phrases among CQs. In the subsequent sections we use the name CQ2SPARQLOWL RULES + NOUN CHUNKING to reference this method.

The task of automatic extraction of Glossary of Terms from textual requirements is also related to software engineering, where the goal is to identify phrases representing names of classes and methods.

Dwarakanath et al. [4] proposed a method extracting such phrases using dependency parsing and filtering. The method is designed to work on a set of declarative sentences and considers only concrete nouns and verbs as candidates for class and method names. In contrast, in the ontology context, abstract nouns, which are nouns representing things that cannot be seen or touched (e.g. education) are good candidates for ontology classes and should not be rejected.

Park et al. [18] proposed GlossEx, a part-of-speech-based system extracting Glossary of Terms in the form of a ranked list of important noun phrases and verbs extracted from a large corpus. This approach generates a single list of important terms from declarative sentences. Ontology requirements however are usually short lists of questions or sentences in which most phrases are related to ontology vocabulary.

\footnotetext{
${ }^{4}$ https://github.com/CQ2SPARQLOWL/Dataset/blob/master/Analysis/pattern_extractor.py

${ }^{5}$ https://spacy.io
} 
Ochodek et al. [17] proposed a method of parsing use cases expressed as sentences to discover transactions. This method uses part-of-speech tags, English Grammar parser and Wordnet [16] to extract actors, predicates, object phrases, validation verbs and object verbs.

\section{Materials}

The dataset used to construct REQTAgger In order to make REQTAGger comparable with alternative automatic Glossary of Terms extraction methods we use CQ2SPARQLOWL dataset, which was used to construct both CRF-BASED TAGGER and CQ2SPARQLOWL RULES + NOUN CHUNKING. We collected all 234 CQs from the dataset. In cases, where a given CQ contains a placeholder and should be materialized, we replaced it with a randomly chosen class fitting the placeholder constraints (e.g.: Is there a mailing list for [this software]? was materialized with Weka piece of software into Is there a mailing list for Weka?.

We used CQ2SPARQLOWL patterns to identify which phrases were considered as domain phrases as entities (tagged in CQ2SPARQLOWL as ECs) or relations (tagged as PCs) to make sure that we define candidates suggestions in the same way. Since the authors of CRF-BASED TAGGER utilized the same process to create their training set, our methods can be fairly compared. There is a possible ambiguity when for example two nouns are separated by an adposition, e.g. meeting in the club. The whole phrase can be considered as a suggestion for a single class/individual, or 'meeting' and 'the club' can be suggested as two different classes/individuals. Both CRF-BASED TAGGER and CQ2SPARQLOWL RULES + NOUN CHUNKING interpret such cases as two separate classes/individuals and we follow that convention.

The evaluation dataset To evaluate the proposed rules, we collected a separate set of requirements coming from the CORAL requirements dataset. We chose all requirements from that dataset from ontologies which are not a part of CQ2SPARQLOWL, to ensure that the evaluation dataset is different than the one used to construct the method. As a result, we collected a large corpora of requirements expressed as CQs and statements. A summary of the dataset size is presented in Table 1.

The evaluation dataset was annotated by an expert who extracted golden standard phrases. For each requirement, lists of candidates for entities (classes or instances) and relations (data and object properties) were extracted separately. As a result, we extracted 1779 phrases, out of which 1364 were categorized as entities and 416 as relations. The detailed summary can be found in Table 2 .

Part-of-speech tagsets selection We have chosen two part-of-speech tagsets available in $\mathrm{SpaCy}^{6}$ NLP tool to verify which one is better suited for the given task:

\footnotetext{
${ }^{6}$ https://spacy.io
} 
Table 1. Evaluaton set extracted from CORAL corpus.

\begin{tabular}{|l|r|r|}
\hline Ontology name & Number of questions & Number of statements \\
\hline VICINITY Core & 58 & 68 \\
\hline VICINITY WOT & 7 & 17 \\
\hline VICINITY WOT mappings & 0 & 15 \\
\hline BTN100 & 77 & 0 \\
\hline SAREF & 1 & 70 \\
\hline SAREF4BLDG & 97 & 1 \\
\hline SAREF4ENVI & 18 & 40 \\
\hline OneM2M & 0 & 58 \\
\hline ODRL & 0 & 12 \\
\hline BTO & 0 & 18 \\
\hline \hline TOTAL & 258 & 299 \\
\hline
\end{tabular}

Table 2. Number of golden standard phrases tagged manually in the evaluation set by an expert.

\begin{tabular}{|l|r|r|}
\hline Questions or Statements & Number of entities & Number of relations \\
\hline Questions & 472 & 89 \\
\hline Statements & 892 & 327 \\
\hline
\end{tabular}


- Universal POS-tags: a coarse-grained part-of-speech tags coming from Universal Dependencies ${ }^{7}$, consisting of 17 distinct labels,

- OntoNotes 5 version of the Penn Treebank POS-tags (further abbreviated as OntoNotes POS-tags): a fine-grained POS-tag set consisting of 53 distinct labels $^{8}$.

\section{Method}

In this section we describe how REQTAGGER works. In the first subsection we introduce the rule language and provide the list of rules we created manually based on CQ2SPARQLOWL dataset. In the second subsection we provide the detailed description of REQTAGGER processing steps. In the third subsection information about the implementation and source code location are presented.

\subsection{Rules}

Rule language The rules we propose are entirely based on POS-tag sequences. Since every token in a tokenized question or statement has exactly one POS-tag assigned, if a rule matches some sequence of POS-tags, it is trivial to identify which tokens they are assigned to.

A pattern to be matched by a rule is a sequence of items. A single item consists of a set of POS-tags expected at a given position, preceeded by optional modifiers indicating how many matches of given POS-tags we expect.

There are 3 modifiers available:

- $\{0+\}$ - meaning that we expect at least zero (the more the better) tokens matching a given POS-tags set.

- $\{1+\}-$ meaning that we expect at least one (the more the better) tokens matching a given POS-tags set.

- $\{1$ ? $\}$ - meaning that we expect at most one tokens matching a given POS-tags set.

Each POS-tag set can consist of a single POS-tag or a list of alternatives separated with | character.

Depending on POS-tag set used, the allowed POS-tag identifiers come from either Universal or OntoNotes based tagset.

An example of a rule using Universal POS-tags is:

$\{0+\}$ ADJ $\{1+\}$ NOUN|PROPN VERB

\footnotetext{
${ }^{7}$ https://universaldependencies.org/u/pos/

${ }^{8}$ https://spacy.io/api/annotation\#pos-en
} 
Table 3. Rules for entities and relations extraction based on Universal POS-tags. The identifiers are described in Table 5.

\begin{tabular}{|l|}
\hline Entities extraction rules \\
\hline 1. $\{0+\}$ ADJ $\{1+\}$ NOUN | PROPN \\
2. $\{0+\}$ ADJ $\{1+\}$ NOUN | PROPN $\{0+\}$ ADJ $\{1+\}$ NOUN | PROPN \\
3. VERB NOUN | PROPN \\
\hline Relations extraction rules \\
\hline 1. $\{0+\}$ PART | VERB | AUX VERB \\
2. $\{0+\}$ VERB ADV | AUX \\
3. $\{0+\}$ PART | VERB | AUX $\{1+\} A U X \mid$ VERB | ADJ | ADV ADP |SCONJ \\
\hline
\end{tabular}

The rule can be interpreted as: Search for a sequence of at least zero adjectives, followed by at least one noun or proper noun, followed by exactly one verb.

Since there are modifiers indicating the allowed number of matches provided, we decided to let the rules work in a greedy way (see Figure 1), thus, the rule:

$\{1+\}$ NOUN

covers as long consecutive sequence of nouns as possible.

Figure 1. If many possible matches can be generated using a single rule, the longest one is returned.

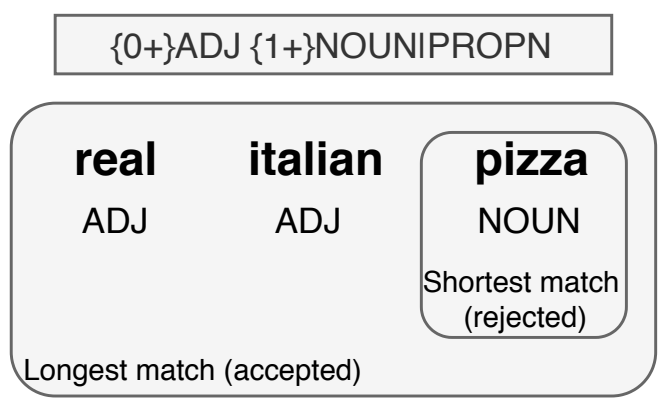

Rules set Based on CQ2SPARQLOWL dataset, we created rules for entities and relations extraction expressed using Universal and OntoNotes POS-tags separately. Our objective was to extract as many expected phrases as possible, and introduce the lowest possible number of wrong suggestions. The rules constructed using Universal POS-tags are listed in Table 3. The rules constructed using OntoNotes POS-tags are listed in Table 4. Table 5 and Table 6 describe POS-tags used in rules using Universal and OntoNotes POS-tags respectively. 
Table 4. Rules for entities and relations extraction based on OntoNotes POS-tags. The identifiers are described in Table 6 .

\begin{tabular}{|c|}
\hline Entities extraction rules \\
\hline $\begin{array}{l}\text { 1. }\{1 \text { ? }\} D T\{0+\} J J|J J S| F W|N N| N N S \mid N N P \text { NN } \mid \text { NNP } \mid \text { NNS } \\
\text { 2. } \mathrm{DT} \quad V B|V B G| V B D|V B Z| V B N\{0+\} N N|N N S| N N P \mid J J \text { NN } \mid \text { NNS } \mid \text { NNP }\end{array}$ \\
\hline Relations extraction rules \\
\hline 1. $\{1+\} \mathrm{JJ}$ IN \\
\hline 2. JJR IN \\
\hline 3. $\mathrm{RB} \mathrm{VBD}$ \\
\hline 4. $\{0+\} \mathrm{MD}\{1+\} \mathrm{VBZ} \mid \mathrm{VBN} \mathrm{TO} \mathrm{VB}$ \\
\hline 5. $\{0+\} \mathrm{MD}$ VB VBN IN \\
\hline 6. $\{1$ ? $\} \mathrm{TO} \mathrm{VB}|\mathrm{VBN}| \mathrm{VBZ} \mid \mathrm{VBP}\{1 ?\} \mathrm{JJ}$ IN \\
\hline 7. $\{0+\} \mathrm{VB} \mid \mathrm{MD}\{0+\} \mathrm{TO} \mathrm{VB}$ \\
\hline 8. $\{1 ?\} \mathrm{TO}\{0+\} \mathrm{VB}|\mathrm{VBP}| \mathrm{VBZ}|\mathrm{VBN}| \mathrm{VBD} \mid \mathrm{VBG}$ \\
\hline$\{1+\}$ RBS $\mid$ VB $|V B Z| V B N|V B D| V B G \mid I N$ RBS | IN \\
\hline 9. $\{1$ ? $\} \mathrm{TO} \mathrm{VB}|\mathrm{VBZ}| \mathrm{VBP}|\mathrm{VBG}| \mathrm{VBD} \mathrm{VB}|\mathrm{VBN}| \mathrm{VBG}|\mathrm{VBG}| \mathrm{VBD}|\mathrm{JJR}| \mathrm{RP}$ \\
\hline 10. VBN VBG \\
\hline
\end{tabular}

Table 5. Descriptions of identifiers used in rules based on Universal POS-tags.

\begin{tabular}{|l|r|r|}
\hline Identifier & description & example \\
\hline ADJ & adjective & blue \\
NOUN & noun & chair \\
PROPN & proper noun & Mike \\
VERB & verb & reading \\
PART & particle & possesive marker's \\
AUX & auxiliary verb & should \\
ADV & adverb & up \\
ADP & adposition & during \\
SCONJ & subordinating conjunction & through \\
\hline
\end{tabular}


Table 6. Descriptions of identifiers used in rules based on OntoNotes POS-tags.

\begin{tabular}{|l|r|r|}
\hline Identifier & description & example \\
\hline VB & verb, base form & be \\
VBZ & verb, 3rd person sing. present & is \\
VBN & verb, past participle & been \\
VBG & verb, gerund/present participle & being \\
VBP & verb, sing. present, non-3d & are \\
RP & particle & give up \\
RB & adverb & good \\
RBS & adverb, superlative & best \\
DT & determiner & the \\
JJ & adjective & blue \\
JJS & adjective, superlative & tallest \\
JJR & adjective, comparative & taller \\
NN & noun, singular or mass & chair \\
NNS & noun plural & chairs \\
NNP & proper noun & Mike \\
IN & preposition, subordinating conjunction & of \\
MD & modal & will \\
\hline
\end{tabular}

\subsection{ReQTAgger Workflow}

In Figure 2 we present the workflow of REQTAGGER, which consists of 3 steps introduced in the subsequent paragraphs. The tagger expects a requirement represented in natural language as an input and transforms it into two sequences of strings: the first listing candidates for entities and the second listing candidates for relations.

Step 1: Rule-based entities and relations extraction Each requirement represented as a string is tokenized and POS-tagged depending on which POS-tag set was selected. Then, rules introduced in Section 4.1 are used to mark spans of POStags matching the rules, so that token sequences associated with matched POS-tag sequences are extracted. Since two types of rules (extracting entities and relations) are used independently, two lists of token sequences are produced.

Step 2: Resolving overlaps It may occur, that two rules detecting entities or relations mark overlapping sequences of tokens in a sense that one of the sequences will be a substring of other sequence, or two sequences will be the same. We filter such cases, so only one (longer if the other match is a substring) phrase is extracted in that situation. An example of this step is presented in Figure 3 (notice that this is a different situation than presented in Figure 1, here matches are generated by different rules). 


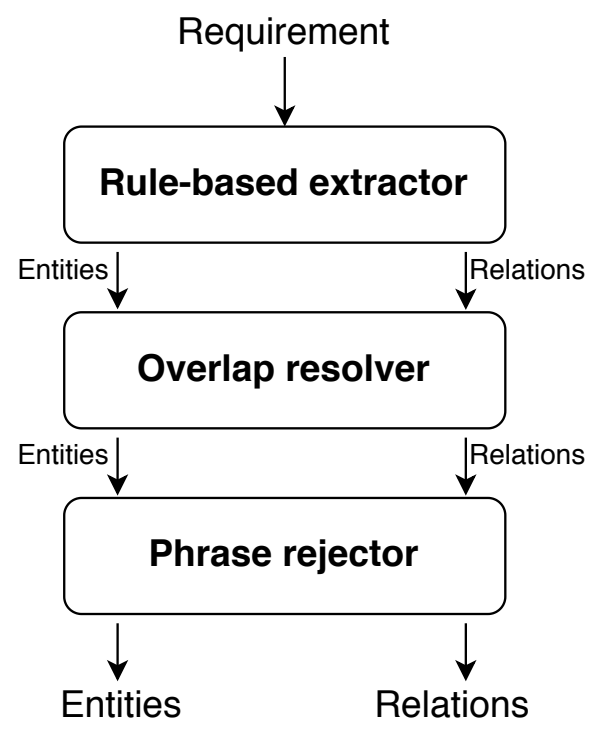

Figure 2. REQTAGGER workflow.

Step 3: Phrase rejection Although some verbs are meaningful candidates for relations (e.g. utilizes, requires), some are not (is, did, has, given). To handle such cases, based on CQ2SPARQLOWL dataset, we curated a list for relation candidates that are extracted using rules, but should be rejected as bad candidates for vocabulary. The list contains the following words and phrases: is, are, was, do, does, did, were, have, had, can, could, regarding, is of, are of, are in, given, is there, has. If an extracted candidate consists of exactly the same sequence of characters as one of the mentioned phrases, it is discarded and not presented to the user. Similarly, the following words: kind, type, category and their variants (with determiners, plurals) are rejected as candidates for entities.

\subsection{Published implementation and source code}

ReQTAgger is implemented in Python and publicly available under MIT license. The source code, the evaluation set tagged with entities and relations to be extracted as well as scripts for evaluation scores calculation are published on GitHub ${ }^{9}$.

\footnotetext{
${ }^{9}$ https://github.com/reqtagger/ReqTagger
} 
Figure 3. If two different rules produce outputs, out of which one is a substring of the other, or the outputs are the same, only one output is presented to the user (the longer one in case of outputs of different lengths).

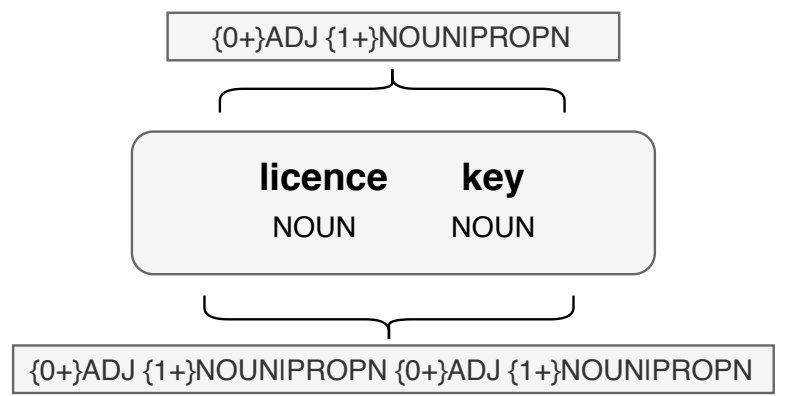

\section{Evaluation}

Given a list of phrases expected to be extracted from each evaluation requirement, we use precision (Equation 1), recall (Equation 2) and $F_{1}$ (Equation 3) to check how many of the suggestions generated by evaluated methods are correct, how many phrases expected to be extracted are generated and what is the harmonic average of precision and recall respectively. In Equations 1, 2, 3, TP (true positives) represents the number of phrases expected to be extracted that were correctly extracted by an evaluated method, FP (false positives) the number of extracted phrases that were not expected to be extracted and $F N$ (false negatives), the number of expected phrases, that were not generated by the evaluated method.

To classify an extracted phrase as TP, the phrase should be the same as one of expected ones. Since there are different approaches to model entities and relations among different ontologies, we still consider two phrases the same if:

- Generated and expected entities differ in presence of an article (a/an/the), thus 'a chair' is considered the same as 'chair'

- Generated and expected relations differ in presence of an auxiliary verb, thus, 'is located at' is considered the same as 'located at'

If a generated phrase is not a $T P$, the $F P$ is generated. If an expected phrase is not generated, the $F N$ is generated.

$$
\begin{gathered}
P=\frac{T P}{T P+F P} \\
R=\frac{T P}{T P+F N} \\
F_{1}=\frac{2 P \cdot R}{P+R}
\end{gathered}
$$


We compare REQTAGGER in two versions: using Universal POS-tag set and OntoNotes POS-tag set with the following alternative methods:

- CQ2SPARQLOWL RULES + NOUN CHUNKING - a method consisting of rules provided by Wisniewski et al. [26] and a noun chunking module from SpaCy.

- CRF-Based tagger (SWO) - a method based on Conditional Random Fields, trained on materialized questions from SWO ontology (subset of

CQ2SPARQLOWL). The training hyperparameters selected are the same as proposed by Wisniewski et al. [27]: L1 regularization $=1$, L2 regularization $=0.001$ and max iterations number $=100$. Also the same features are used to represent each token:

- string - lowercased token

- boolean flag - is the token an auxiliary verb?

- string - last 3 characters of the token

- boolean flag - does the token start with capital letter?

- boolean flag - does the token contain multiple capital letters?

- number - how many verbs are there in a requirement?

- boolean flag - is the token a digit?

- string - the POS-tag of the token (OntoNotes tag set)

- string - the first two characters of the POS-tag of the token

- string - dependency tree label assigned to the token

- string - dependency tree label of the parent of the token

Features from context tokens ( 2 previous, 2 subsequent) are concatenated to the current token features list to encode the textual context.

- CRF-BAsed tagger (CQ2SPARQLOWL) - a method analogous to CRFBASED TAGGER ON SWO trained on all materialized requirements from CQ2SPARQLOWL.

We split the evaluation to observe what are the scores calculated over entities and relations, as well as questions and statements separately. The scores for questions and statements are aggregated in Table 7 and Table 8 respectively. The average $F_{1}$ values, aggregated over $F_{1}$ scores of entities and relations from questions and statements, are presented in Table 9 .

\section{Error analysis}

In this section we discuss the most interesting errors generated by REQTAGGER and analyze their causes. We split the analysis into two parts: Entities and Relations, since both are generated using different rules. Because the best quality is achieved using OntoNotes POS-tag set, we focus our analysis on this approach, treating it as the default tagset of REQTAGGER. 
Table 7. Methods scored using precision, recall and $F_{1}$, calculated over evaluation questions. The scores are calculated separately over entities and relations. The highest scores are marked with a bold font.

\begin{tabular}{|l|r|r|r||r|r|r|r|}
\hline Scenario & \multicolumn{3}{|c||}{ Scores entities } & \multicolumn{3}{|c|}{ Scores relations } \\
& $\mathrm{P}$ & $\mathrm{R}$ & $F_{1}$ & $\mathrm{P}$ & $\mathrm{R}$ & $F_{1}$ \\
\hline REQTAGger (ONTONOtES POS-TAGs) & $\mathbf{0 . 9 6 8}$ & $\mathbf{0 . 9 7 2}$ & $\mathbf{0 . 9 7}$ & 0.835 & $\mathbf{0 . 8 5 4}$ & $\mathbf{0 . 8 4 4}$ \\
\hline REQTAGGER (UNIVERSAL POS-TAGS) & 0.958 & 0.964 & 0.961 & 0.773 & 0.843 & 0.806 \\
\hline CQ2SPARQLOWL RULES + NOUN CHUNKING & 0.645 & 0.778 & 0.705 & 0.788 & 0.753 & 0.77 \\
\hline CRF-BASED TAGGER (SWO) & 0.872 & 0.78 & 0.823 & 0.875 & 0.236 & 0.372 \\
\hline CRF-BASED TAGGER (CQ2SPARQLOWL) & 0.898 & 0.824 & 0.86 & $\mathbf{0 . 9 2 3}$ & 0.27 & 0.417 \\
\hline
\end{tabular}

Table 8. Methods scored using precision, recall and $F_{1}$, calculated over evaluation statements. The scores are calculated separately over entities and relations. The highest scores are marked with a bold font.

\begin{tabular}{|l|r|r|r||r|r|r|}
\hline Scenario & \multicolumn{3}{|c||}{ Scores entities } & \multicolumn{3}{c|}{ Scores relations } \\
& $\mathrm{P}$ & $\mathrm{R}$ & $F_{1}$ & $\mathrm{P}$ & $\mathrm{R}$ & $F_{1}$ \\
\hline REQTAGGER (OnTONotes POS-TAGs) & $\mathbf{0 . 8 9 1}$ & $\mathbf{0 . 8 8 8}$ & $\mathbf{0 . 8 8 9}$ & $\mathbf{0 . 7 4 9}$ & $\mathbf{0 . 8 6 9}$ & $\mathbf{0 . 8 0 5}$ \\
\hline REQTAGGER (UNIVERSAL POS-TAGS) & 0.86 & 0.861 & 0.861 & 0.718 & 0.865 & 0.785 \\
\hline CQ2SPARQLOWL RULES + ChUNKING & 0.837 & 0.83 & 0.833 & 0.722 & 0.706 & 0.714 \\
\hline CRF-BASED TAGGER (SWO) & 0.544 & 0.355 & 0.43 & 0.678 & 0.18 & 0.285 \\
\hline CRF-BASED TAGGER (CQ2SPARQLOWL) & 0.604 & 0.401 & 0.482 & 0.687 & 0.174 & 0.278 \\
\hline
\end{tabular}

\subsection{Entities}

many + noun In examples from CORAL: How many rivers flow into the sea or ocean zzz? and How many organizations can have a partnership?, many rivers and many organizations are generated instead of rivers and organizations. These errors are due to POS-tag-based nature of REQTAGGER. In both cases many is tagged as an adjective $(\mathrm{JJ})$, thus, we cannot differentiate between many rivers and blue rivers.

Expected verb with 'ing' suffix as a part of an expected entity In the CQ: Which properties have a protective device tripping unit?, the phrase a protective device tripping unit is expected to be a candidate for an entity. Our rules cannot produce such a result, showing two candidates instead: protective device and unit. The reason for that kind of error is the lack of a rule allowing gerund (verb used as a noun) between nouns defined. Using OntoNotes POS-tag set we can fix this problem by adding a rule allowing a gerund (VBG POS-tag) to be located between nouns. Using Universal POS-tag set such fix is not possible, since there is only one tag for all forms of verbs. Allowing all kinds of verbs to be placed between nouns would result in suggesting multiple wrong suggestions (e.g. Mark likes Jane as a single entity suggestion).

gerund + noun In the CQ: Which are the FFCC stations that are stopping places and which are the FFCC stations that have passenger traffic?, 
Table 9. Aggregated $F_{1}$ scores. Each score is the arithmetic mean of $F_{1}$ scores for entities and relations of questions and statements.

\begin{tabular}{|l|r|}
\hline Method & Average F1 score \\
\hline REQTAGger (ONTONotes POS-TAGS) & $\mathbf{0 . 8 7 7}$ \\
\hline REQTAGGER (UNIVERSAL POS-TAGS) & 0.853 \\
\hline CQ2SPARQLOWL RULES + CHUNKING & 0.756 \\
\hline CRF-BASED TAGGER ON CQ2SPARQLOWL & 0.509 \\
\hline CRF-BASED TAGGER ON SWO & 0.478 \\
\hline
\end{tabular}

stopping places, which is a sequence of a verb with 'ing' suffix followed by a plural form of a noun is not extracted. Instead only places is generated. Although, similarly to a previous example a rule can be added, such a rule may generate multiple false positives. Imagine a CQ: What is stopping Mark from moving abroad?, in that case is stopping should be considered as a relation rather than considering stopping Mark as an entity suggestion, even if the same sequence of gerund + noun is observed.

POS-tagger failure In cases such as: Which properties does a weight scale affect, affect is tagged as a noun instead of a verb, thus a weight scale affect is generated as an entity suggestion.

\subsection{Relations}

verb in past tense + noun In cases such as What is a cooled beam?, the word cooled is suggested to be a relation. We think that cooled should be a part of an a cooled beam entity suggestion. It cannot be easily fixed, since in multiple cases the current solution works better. Adding a rule matching verb in past tense followed by a noun would generate multiple errors (e.g. met Mark would be extracted from I met Mark).

Rejected phrase which should not be rejected In cases like Which historic places are in a county? the phrase are in can be considered as a good candidate for a relation. However, the analysis made on CQ2SPARQLOWL led us to a decision to add such phrase to the list of rejected phrases, so even if this phrase is extracted with rules, it is discarded.

Incomplete relation In a CQ from CORAL Which villages does the road go through? instead of a full go through relation, only go is extracted. This kind of error can be easily fixed by adding appropriate rule, since the pattern was not observed in CQ2SPARQLOWL dataset. 
POS-tagger failure In the statement IoT gateways expose endpoints. the POS-tagger assigns the word gateways a VBZ POS-tag instead of NNS, thus, gateways expose instead of expose is considered as a relation suggestion.

long relation names Among sentences, we observed that long relations like needed to be executed before in a CQ: A mapping might be needed to be executed before another mapping. are not extracted properly. Instead two suggestions needed to be and to be executed before are generated. Errors of this kind are caused by the fact that the method is constructed on CQs only, where long relation names are not observed. A rule solving that kind of an error can be easily added.

\section{Discussion}

The scores presented in Table 7, Table 8 and Table 9 can be discussed from various perspectives:

Global (best scores perspective) In 11 out of 12 cases, REQTAGGER generated better results than other systems. The only case it was worse was extraction of relations on questions, where the CRF-BASED TAGGER outperformed REQTAGGER in terms of precision. However, although CRF-BASED TAGGER generated high quality relations, very low recall score suggests that majority of expected relations were not generated by the method. Despite REQTAGGER outperforms alternatives using either Universal or OntoNotes POS-tags, using OntoNotes may be recommended, since in every evaluation scenario OntoNotes-based rules provide better quality. The reason for such observation is the fine-grained part-of-speech tagset provided in OntoNotes, which allows to generate more precise rules. The scores from Table 9 provide the positive answer to RQ1: REQTAGGER outperforms other methods, considering the overall quality measured using $F_{1}$ score.

Questions versus Statements Each tested method scored considerably higher on questions rather than statements. The reason for that situation is the fact that both rules and the CRF-BASED TAGGER were constructed over a dataset consisting of questions only. It is an interesting fact that both REQTAGGER and CQ2SPARQLOWL RULES + NOUN CHUNKING generate good scores on statements, while a CRF-BASED TAGGER scores very low. We hypothesize that this situation may be a result of the nature of the model - a CRF-BASED MODEL relies heavily on the context of a given token, which is different (e.g. regarding grammar) in statements than in questions. The scores provided in Table 8 provide the positive answer to RQ2: REQTAGger generalize from questions to statements.

Entities versus Relations In almost every evaluation scenario, the scores obtained on entities are higher than on relations. 
Figure 4. A dependency parse tree constructed over a sample CQ from CQ2SPARQLOWL dataset showing that Which software is a single noun chunk, thus CQ2SPARQLOWL RULES + NOUN CHUNKING consider it as a suggestion for a class name. Arrows point from head to children nodes.

Dependency tree

POS tags (Universal)

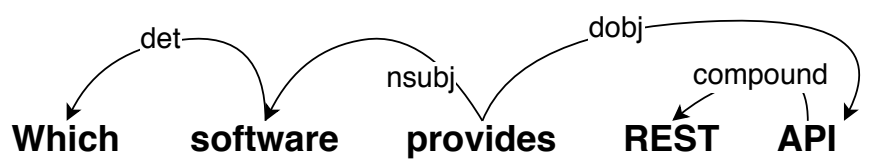

DET

NOUN

VERB

PROPN NOUN

The reason is that candidates for entities are well defined, while candidates for relations are more vague. When creating rules based on CQ2SPARQLOWL dataset, we decided that is in is not a good candidate for a relation. When evaluating on the evaluation dataset, we noticed cases in which considering it as a relation can be reasonable.

The only case in which entities score lower than relations are questions processed by CQ2SPARQLOWL RULES + NOUN CHUNKING, which used noun chunking to extract entities. The analysis of generated outputs revealed the source of the problem: the noun chunker used for entities extraction searches for phrases in which a noun is the head of the phrase in the dependency tree constructed over input question or sentence. It is a frequent scenario in which after 'Wh-' word used at the beginning of the question a noun is placed. In that case, the 'Wh-' word is a child of the noun in a dependency tree. An example of that situation can be seen in Figure 4, where the token software is the parent of the word Which, thus, the noun chunker proposes the whole phrase Which software as a suggestion for an entity, which is obviously wrong.

It is worth noting that REQTAGGER achieved almost perfect quality on extraction of entities from questions using both Universal and OntoNotes POS-tag sets.

The overall quality of extraction presented in Table 9 shows that rule-based methods significaly outperformed the CRF-BASED TAGGER. Although adding new CQs to the training dataset for CRF-BASED TAGGER increased the aggregated $F_{1}$ score by $6.05 \%$ it is still far behind any rule-based method.

\section{Method Limitations}

Although the quality of our model is very good, there are several limitations due to our decision to use POS-tags as the core of REQTAGGER:

- The rules can only extract consecutive sequences of tokens. Consider the following CQ: Do you prefer white or brown rice?. In that case it is reasonable to extract two suggestions: brown rice and white rice. With REQTAGGER 
only the first one can be extracted. The second phrase cannot be extracted, since there are words between white and rice which cannot be omitted using the method.

- Since rules are expressed as sequences of POS-tags only, they are not aware of the meaning of extracted phrases. It is important to maintain a list of rejected phrases, unlikely to be represented in an ontology as classes/individuals or properties. Although our rejection phrases generalize well, it may be a case that some phrases will have to be added.

- REQTAGger is useful for extraction and deciding whether something looks like an entity or a relation. We have to remember that it cannot dive deeper and determine whether a given sequence of tokens should be considered as a class name rather than an individual, or a relation is more likely to be an object property rather than data property. These are design choices made by the ontology engineer.

\section{Conclusions}

Glossary of Terms extraction is an important step of ontology engineering methodologies such as NeON methodology. Although an automatic machine learning-based method was recently proposed, we show that a rule-based method expressing rules in terms of POS-tags can be a better choice for that task, providing a significantly higher quality of the generated outputs than the existing CRF-BASED TAGGER. We show that even if machine learning-based and rule-based methods were constructed over a set of questions only, rule-based methods perform really well, as compared to a machine learning-based method in the task of extracting entities and relations from statements. The rule-based methods can be easily interpreted and changed, so solving errors is much easier than in machine learning-based methods. We show that REQTAGGER scores higher than an alternative rule-based method (CQ2SPARQLOWL RULES + NOUN CHUNKING), because REQTAGGER is designed to provide the best quality of extracted Glossary of Terms suggestions, while CQ2SPARQLOWL RULES + NOUN CHUNKING is designed to solve a related problem of generating domainagnostic CQ patterns. REQTAGGER provide a simple rules language, making it easy to extend the list of predefined rules. Because REQTAGger is available as a Python script, it can be easily run and modified. We recommend REQTAGGER as a method for automatic extraction of Glossary of Terms from ontology requirements.

\section{References}

[1] Antoniou G. and Van Harmelen F. Web ontology language: Owl. In Handbook on ontologies, pages 67-92. Springer, 2004. 
[2] Bezerra C., Santana F., and Freitas F. Cqchecker: A tool to check ontologies in owl-dl using competency questions written in controlled natural language. Learning and Nonlinear Models, 12:115-129, 2014.

[3] del Carmen Suárez-Figueroa M., de Cea G. A., Buil C., Dellschaft K., FernándezLópez M., García A., Gómez-Pérez A., Herrero G., Montiel-Ponsoda E., Sabou M., Villazon-Terrazas B., and Yufei Z. D5.4.1 neon methodology for building contextualized ontology networks, Feb. 2008.

[4] Dwarakanath A., Ramnani R. R., and Sengupta S. Automatic extraction of glossary terms from natural language requirements. In 21st IEEE International Requirements Engineering Conference, RE 2013, Rio de Janeiro-RJ, Brazil, July 15-19, 2013, pages 314-319. IEEE Computer Society, 2013.

[5] Fernández-Izquierdo A., Poveda-Villalón M., and García-Castro R. CORAL: A corpus of ontological requirements annotated with lexico-syntactic patterns. In ESWC, 2019.

[6] Fernandez-Lopez M., Gomez-Perez A., and Juristo N. Methontology: from ontological art towards ontological engineering. In Proceedings of the AAAI97 Spring Symposium, pages 33-40, Stanford, USA, March 1997.

[7] Grishman R. Information extraction: Techniques and challenges. In International summer school on information extraction, pages 10-27. Springer, 1997.

[8] Gruninger M. Methodology for the design and evaluation of ontologies. In IJCAI 1995, 1995.

[9] Huang Z., Xu W., and Yu K. Bidirectional lstm-crf models for sequence tagging. arXiv preprint arXiv:1508.01991, 2015.

[10] Keet C. M., Mahlaza Z., and Antia M.-J. Claro: a data-driven cnl for specifying competency questions. arXiv preprint arXiv:1907.07378, 2019.

[11] Lafferty J. D., McCallum A., and Pereira F. C. N. Conditional random fields: Probabilistic models for segmenting and labeling sequence data. In Proceedings of the Eighteenth International Conference on Machine Learning, ICML '01, page 282-289, San Francisco, CA, USA, 2001. Morgan Kaufmann Publishers Inc.

[12] Lawrynowicz A. and Keet C. M. The TDDonto tool for test-driven development of DL knowledge bases. In Lenzerini M. and Peñaloza R., editors, Description Logics, volume 1577 of CEUR Workshop Proceedings. CEUR-WS.org, 2016.

[13] Lenat D. B. and Guha R. V. Building Large Knowledge-Based Systems; Representation and Inference in the Cyc Project. Addison-Wesley Longman Publishing Co., Inc., USA, 1st edition, 1989.

[14] Ling X. and Weld D. S. Fine-grained entity recognition. In Proceedings of the Twenty-Sixth AAAI Conference on Artificial Intelligence, AAAI'12, page 94-100. AAAI Press, 2012. 
[15] Malone J., Brown A., Lister A., Ison J., Hull D., Parkinson H., and Stevens R. The software ontology (SWO): A resource for reproducibility in biomedical data analysis, curation and digital preservation. Journal of biomedical semantics, 5:25, 062014.

[16] Miller G. A. WordNet: A lexical database for english. Commun. ACM, 38(11):3941, 1995.

[17] Ochodek M. and Nawrocki J. R. Automatic transactions identification in use cases. In Meyer B., Nawrocki J. R., and Walter B., editors, Balancing Agility and Formalism in Software Engineering, Second IFIP TC 2 Central and East European Conference on Software Engineering Techniques, CEE-SET 2007, Poznan, Poland, October 10-12, 200\%, Revised Selected Papers, volume 5082 of Lecture Notes in Computer Science, pages 55-68. Springer, 2007.

[18] Park Y., Byrd R. J., and Boguraev B. Automatic glossary extraction: Beyond terminology identification. In 19th International Conference on Computational Linguistics, COLING 2002, Howard International House and Academia Sinica, Taipei, Taiwan, August 24 - September 1, 2002, 2002.

[19] Petrucci G., Ghidini C., and Rospocher M. Ontology learning in the deep. In Knowledge Engineering and Knowledge Management - 20th International Conference, EKAW 2016, Bologna, Italy, November 19-23, 2016, Proceedings, pages 480-495, 2016.

[20] Potoniec J., Wisniewski D., Lawrynowicz A., and Keet C. M. Dataset of ontology competency questions to SPARQL-OWL queries translations. Data in Brief, 29, 2020 .

[21] Ren Y., Parvizi A., Mellish C., Pan J. Z., van Deemter K., and Stevens R. Towards competency question-driven ontology authoring. In Presutti V., d'Amato C., Gandon F., d'Aquin M., Staab S., and Tordai A., editors, The Semantic Web: Trends and Challenges, pages 752-767, Cham, 2014. Springer International Publishing.

[22] Suárez-Figueroa M. C., Gómez-Pérez A., and Fernández-López M. The NeOn Methodology for Ontology Engineering, pages 9-34. Springer Berlin Heidelberg, Berlin, Heidelberg, 2012.

[23] Sure Y., Staab S., and Studer R. On-To-Knowledge Methodology (OTKM), pages 117-132. Springer Berlin Heidelberg, Berlin, Heidelberg, 2004.

[24] Uschold M. and King M. Towards a methodology for building ontologies. In In Workshop on Basic Ontological Issues in Knowledge Sharing, held in conjunction with IJCAI-95, 1995.

[25] Wisniewski D. Automatic translation of competency questions into SPARQLOWL queries. In Companion Proceedings of the The Web Conference 2018, WWW '18, page 855-859, Republic and Canton of Geneva, CHE, 2018. International World Wide Web Conferences Steering Committee. 
[26] Wisniewski D. et al. Analysis of ontology competency questions and their formalizations in SPARQL-OWL. JWS, 59, 2019.

[27] Wisniewski D. and Ławrynowicz A. A tagger for glossary of terms extraction from ontology competency questions. In Proc. of ESWC, Satellite Events, pages 181-185. Springer, 2019.

[28] Wisniewski D., Potoniec J., and Lawrynowicz A. BigCQ: A large-scale synthetic dataset of competency question patterns formalized into SPARQL-OWL query templates. CoRR, abs/2105.09574, 2021.

Received 0\%.04.2021, Accepted 10.09.2021 